\title{
Efficient and accurate computation of base generation allele frequencies
}

\author{
M. N. Aldridge, ${ }^{*}$ J. Vandenplas, and M. P. L. Calus \\ Wageningen University \& Research, Animal Breeding and Genomics, 6700AH Wageningen, the Netherlands
}

\begin{abstract}
Allele frequencies are used for several aspects of genomic prediction, with the assumption that these are equal to the allele frequency in the base generation of the pedigree. The current standard method, however, calculates allele frequencies from the current genotyped population. We compared the current standard method with BLUP and general least squares (GLS) methods explicitly targeting the base population to determine whether there is a more accurate and still efficient method of calculating allele frequencies that better represents the base generation. A data set based on a typical dairy population was simulated for 325,266 animals; the last 100,078 animals in generations 9 to 12 of the population were genotyped, with 1,670 SNP markers. For the BLUP method, several SNP genotypes were analyzed with a multitrait model by assuming a heritability of 0.99 and no genetic correlation among them. This method was limited by the time required for each BLUP to converge (approximately $6 \mathrm{~min}$ per BLUP run of 15 SNP). The GLS method had 2 implementations. The first implementation, using imputation on the fly and multiplication of sparse matrices, was very efficient and required just $49 \mathrm{~s}$ and $1.3 \mathrm{~GB}$ of random access memory. The second implementation, using a dense full $\mathbf{A}_{22}^{-1}$ matrix, was very inefficient and required more than $1 \mathrm{~d}$ of wall clock time and more than 118.2 GB of random access memory. When no selection was considered in the simulations, all methods predicted equally well. When selection was introduced, higher correlations between the estimated allele frequency and known base generation allele frequency were observed for BLUP $(0.96 \pm 0.01)$ and GLS $(0.97$ $\pm 0.01)$ compared with the current standard method $(0.87 \pm 0.01)$. The GLS method decreased in accuracy when introducing incomplete pedigree, with $25 \%$ of sires in the first 5 generations randomly replaced as unknown to erroneously identify founder animals (0.93
\end{abstract}

Received June 22, 2018.

Accepted October 1, 2018.

*Corresponding author: michael.aldridge@wur.nl $\pm 0.01)$ and a further decrease for 8 generations (0.91 $\pm 0.01)$. There was no change in accuracy when introducing $5 \%$ genotyping errors $(0.97 \pm 0.01), 5 \%$ missing genotypes $(0.97 \pm 0.01)$, or both $5 \%$ genotyping errors and missing genotypes $(0.97 \pm 0.01)$. The GLS method provided the most accurate estimates of base generation allele frequency and was only slightly slower compared with the current method. The efficient implementation of the GLS method, therefore, is very well suited for practical application and is recommended for implementation.

Key words: general least squares, best linear unbiased prediction, dairy cattle

\section{INTRODUCTION}

Allele frequencies are required for several processes in genomic prediction. The assumption for these processes is that the allele frequencies used are equal to the allele frequency of the base generation, commonly defined as the pedigree founders. For multistep genomic evaluations, allele frequencies are used for the computation of model-based reliabilities of direct genomic values (VanRaden, 2008). However, VanRaden (2008) showed that there was limited effect on reliabilities of genomic predictions when using base generation or estimated allele frequencies. For single-step genomic BLUP, allele frequencies are used for the computation of genomic relationships (Aguilar et al., 2010; Christensen and Lund, 2010). The compatibility between pedigree and genomic relationships is an important issue in single-step genomic BLUP, as differences in the bases of both matrices may lead to bias of the predictions and reduce their accuracy. This possible bias can be overcome by making adjustments to the genomic relationships (Vitezica et al., 2011; Christensen, 2012; Gao et al., 2012). Using base generation allele frequencies to compute the genomic relationships is another possible approach toward making pedigree and genomic relationships compatible. For estimating relationships among metafounders (pseudoindividuals used as founders in the pedigree, with an unknown sire and dam), the computation is based on the variance of the base generation allele frequencies (Legarra et al., 2015), so 
estimating base generation allele frequencies accurately is essential for this process. However, it is standard practice to use allele frequencies calculated from the current genotyped population because of the ease of computation.

An accurate and computationally efficient method of estimating base generation allele frequencies is desirable to replace the current standard method based on the currently genotyped population. Two methods have been proposed to explicitly estimate the base generation allele frequencies. The first method was to run, for each SNP, a BLUP in which the heritability was close to 1 (e.g., 0.99 or smaller; Gengler et al., 2007). The second method was, for each SNP, a general least squares (GLS) estimator using either sparse or dense matrices for the computation of the inverse of pedigree relationship submatrices (McPeek et al., 2004; Garcia-Baccino et al., 2017; Strandén et al., 2017). The BLUP and GLS methods are expected to be very similar because both use pedigree information, but we did not expect them to be exactly the same, although theoretically equivalent (e.g., Henderson, 1984; Mrode, 2005; Garcia-Baccino et al., 2017) differences between estimates of BLUP and GLS methods could be due to the heritability different from 1 and the iterative solver used in the BLUP method. The objective of this study was to determine the most efficient and accurate method for estimating base generation allele frequencies when different scenarios likely to occur in real data are considered, including missing genotypes, genotyping errors, and incomplete pedigree. We explored alternative implementations to improve the computational efficiency, with a multitrait model for BLUP rather than the previously proposed single-trait model, such that these strategies could be applied with currently available and routinely used software.

\section{MATERIALS AND METHODS}

To achieve our objective, data sets were simulated with a typical Holstein-like dairy population using QMSim (Sargolzaei and Schenkel, 2009). Each data set was simulated with a historical population of 100,000 animals, decreasing to 500 animals over 2,000 generations, and then rapidly increasing to 25,000 animals over 10 generations; this was to establish linkage disequilibrium in the base generation (average $\mathrm{R}^{2}$ between adjacent markers $=0.41$ ). The founder population and base generation for which the allele frequencies were to be estimated consisted of 24,970 females and 30 sires, selected from the final historical generation. The population structure of the historical and founder population was selected to achieve an effective population size of approximately 100. The following 12 generations had a mutation rate of $2.5 \times 10^{-5}$ (same mutation rate as the historical population) to ensure that enough segregating markers in the final generations (Daetwyler et al., 2013), random selections, random matings, and the same sex proportions in the founder population were maintained. The resulting pedigree included a total of 325,266 animals across 12 generations. This base simulation had no selection and was used as a control. Generations 9 to 12 were fully genotyped, which included 100,078 animals. The genotyping included 1,670 SNP with 250 QTL affecting the trait, with a uniform distribution of allele frequencies in the base generation. Single nucleotide polymorphisms and QTL were randomly positioned across 10 chromosomes, and each chromosome was $100 \mathrm{~cm}$ in length. The number of markers was chosen to be similar to that in the additional simulations more likely to occur in reality.

Seven additional data sets were simulated using the same historical and founder population structure as the base simulation but with selection included; depending on the scenario, errors or missing data were introduced to mimic reality (Table 1). All additional data sets used the base simulation, with selection for the last 12 generations based on high EBV obtained with BLUP and considering the true additive genetic variance rather than randomly, and the 1,670 SNP were positioned on a single chromosome of $100 \mathrm{~cm}$. The number of markers was selected for scaling to $1,670 \mathrm{SNP}$ on each of 30 chromosomes to be representative of a commercial $50 \mathrm{~K}$ chipset. In the data sets with selection, only a single chromosome was simulated to achieve a strong effect of selection on the change in allele frequency within a limited number of generations, illustrated by the allele frequency change between the base and the last genotyped generation (Figure 1). In the first data set with selection, it was assumed that the pedigree and all genotypes were known without error. The second data set had an incomplete pedigree, created by randomly replacing $25 \%$ of sires in generations 1 to 5 as unknown parents; this was to replicate a situation in which pedigree records are lost and unknown sires are erroneously identified as base animals. The third data set included extending the number of generations, which randomly replaced $25 \%$ of sires as unknown parents up to generation 8. In the fourth data set, genotyping errors were simulated, where a genotype was replaced by 2 randomly sampled alleles at a rate of $5 \%$. The fifth data simulation randomly introduced missing genotypes at a rate of $5 \%$. The sixth data set included both the $5 \%$ erroneous genotypes and $5 \%$ missing genotypes. Finally, a 50K SNP data set was simulated with 30 chromosomes each, with 1,670 SNP randomly positioned. 
In all scenarios a single data set was simulated, where the results were evaluated across the 1,670 SNP. Thus, the 1,670 SNP served as replicates across which the results were evaluated. To evaluate whether the dependency between SNP may have affected the averaged results, we also selected a subset of SNP including every 50th SNP and evaluated results for those separately. The average correlation between these $33 \mathrm{SNP}$ was 0.03 and, thus, these 33 SNP were considered to be independent of each other.

The current standard method for calculating the allele frequencies of the genotyped population to be used as base generation allele frequencies was implemented with a Fortran program we developed, hereafter referred to as the "current method." The frequency of allele 1 of the $i$ th SNP, $p_{i}$, was computed as follows:

$$
p_{i}=\frac{n_{1}}{2 n}
$$

where $n_{1}$ is the number of occurrences for allele 1 and $n$ is the total number of alleles. Another implementation was made in which instead of using all genotyped animals, only the oldest genotyped generation was used, assuming that it is a better representation of the base generation because it is more closely connected.

The BLUP method involved evaluating the genotypes of each of the SNP as a phenotype in a BLUP model with the software MiXBLUP (Ten Napel et al., 2017). For each SNP the heritability was set to 0.99 following Gengler et al. (2007). To speed up the analyses, multiple SNP were analyzed simultaneously by the means of a multitrait model with zero genetic correlations among SNP. To determine the optimum number of SNP to be included in each run, a series of analyses were run with the number increasing from 1 to 60 in increments of 5 . Based on the results of these analyses (Figure 2), the final BLUP analysis consisted of 111 BLUP runs of 15 SNP and 1 run of 5 SNP, all performed in parallel. The MiXBLUP convergence criterion for the preconditioned conjugate gradient method was $1.0 \times 10^{-12}$. The base generation allele frequency was estimated for each SNP as $\hat{\mu} / 2$, where $\hat{\mu}$ was the estimate of the general mean of the model. Simulated missing genotypes were considered as missing phenotypes in the analysis.

The GLS equivalent uses the method proposed by McPeek et al. (2004) and implemented by Strandén et al. (2017) and Garcia-Baccino et al. (2017) in which for the $i$ th SNP

$$
\hat{\mu}_{i}=\left(\mathbf{1}^{\prime} \mathbf{A}_{22}^{-1} \mathbf{1}\right)^{-1} \mathbf{1}^{\prime} \mathbf{A}_{22}^{-1} \mathbf{z}_{\mathrm{i}}
$$

where $\mathbf{1}$ is a vector of ones, $\mathbf{A}_{22}^{-1}$ is the inverse pedigree relationship matrix of genotyped animals, and $\mathbf{z}_{\mathrm{i}}$ is a vector of genotypes coded as 0,1 , and 2 . Two implementations of this method were made. Our first implementation, referred to as "GLS_Sparse," was similar to that of Strandén et al. (2017) in the sense that the vector $\mathbf{t}=\mathbf{A}_{22}^{-1} 1$ was first computed as a multiplication of sparse matrices by the vector $\mathbf{1}$, followed by the trivial computation of the scalar $\alpha=\left(\mathbf{1}^{\prime} \mathbf{A}_{22}^{-1} \mathbf{1}\right)^{-1}=\left(\mathbf{1}^{\prime} \mathbf{t}\right)^{-1}$ and by the multiplication of a matrix and vector - that is, $\hat{\mu}=\alpha \mathbf{t}^{\prime} \mathbf{Z}$. The vector $\mathbf{t}$ can be efficiently computed as follows (Strandén et al., 2017):

$$
\boldsymbol{t}=\mathbf{A}_{22}^{-1} \mathbf{1}=\left(\left[\mathbf{A}^{22} \mathbf{1}\right]-\left\{\mathbf{A}^{21}\left[\left(\mathbf{A}^{11}\right)^{-1}\left(\mathbf{A}^{12} \mathbf{1}\right)\right]\right\}\right)
$$

where $\mathbf{A}^{i j}$ are submatrices of $\mathbf{A}^{-1}$ (for $i$ and $j$, a value of 1 denotes nongenotyped animals and a value of 2 denotes genotyped animals), and the brackets indicate the order of the matrix-vector operations.

In our implementation, Math Kernel Library (MKL) subroutines were used for the matrix-vector multiplications, and Intel MKL-Pardiso (Schenk et al., 2001) was used to compute $\mathbf{x}=\left(\mathbf{A}^{11}\right)^{-1}\left[\mathbf{A}^{12} \mathbf{1}\right]=\left(\mathbf{A}^{11}\right)^{-1} \mathbf{v}$ by solving $\mathbf{A}^{11} \mathbf{x}=\mathbf{v}$. In GLS_Sparse, missing genotypes were replaced with the current genotype mean, computed across all animals with observed genotype for this locus. The second implementation of the GLS

Table 1. Summary of the structure and errors for the different data simulations

\begin{tabular}{llll}
\hline Data set & Chromosomes & Selection & Data error \\
\hline Base simulation & 10 & No selection & No errors \\
Base simulation & 1 & High EBV & No errors \\
Incomplete pedigree & 1 & High EBV & $25 \%$ of sires in generation 1 to 5 are randomly replaced as unknown \\
Incomplete pedigree & 1 & High EBV & $25 \%$ of sires in generation 1 to 8 are randomly replaced as unknown \\
Genotyping errors & 1 & High EBV & $5 \%$ of genotypes are replaced by randomly sampled alleles \\
Missing genotypes & 1 & High EBV & $5 \%$ of genotypes are randomly replaced as missing \\
Errors and missing & 1 & High EBV & Both 5\% genotyping errors and missing genotypes \\
50K SNP & 30 & No selection & No errors \\
\hline
\end{tabular}


method instead calculates the full $\mathbf{A}_{22}^{-1}$ directly using Calc_grm (Calus and Vandenplas, 2016), hereafter referred to as "GLS_Full." This approach may mimic an approach in which a user would use available software.
All computations were run on a high-performance cluster (HPC). The HPC was designed with 48 nodes: 16 cores, 64-GB memory, Intel Xeon, and $2.2 \mathrm{GHz}$. A single thread was used for the current, BLUP, and
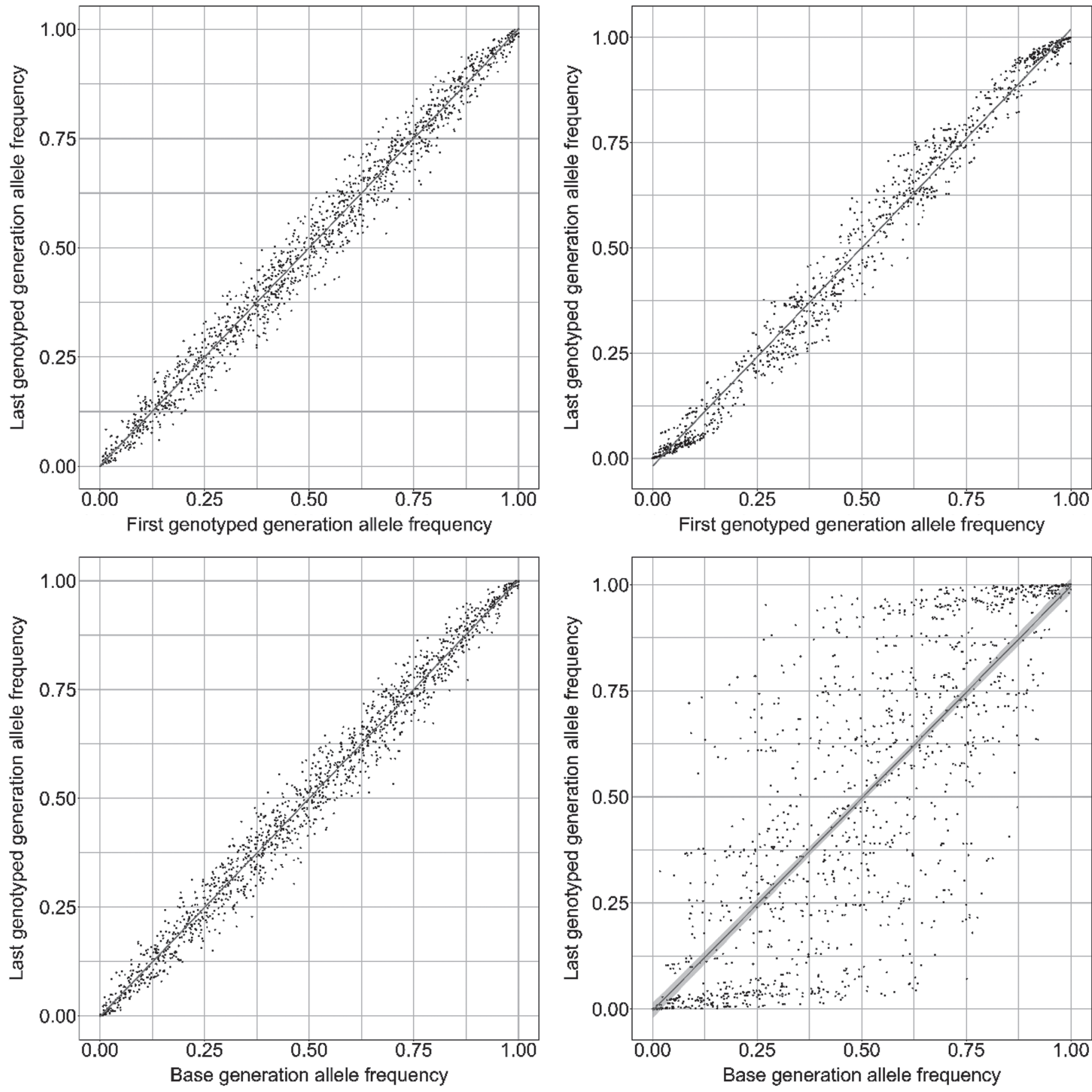

Figure 1. Change in allele frequency between generations 9 and 12 for the base simulation without selection (top left) and the base simulation with selection (top right). Change in allele frequency between generations 0 and 12 for the base simulation without selection (bottom left) and the base simulation with selection (bottom right). 


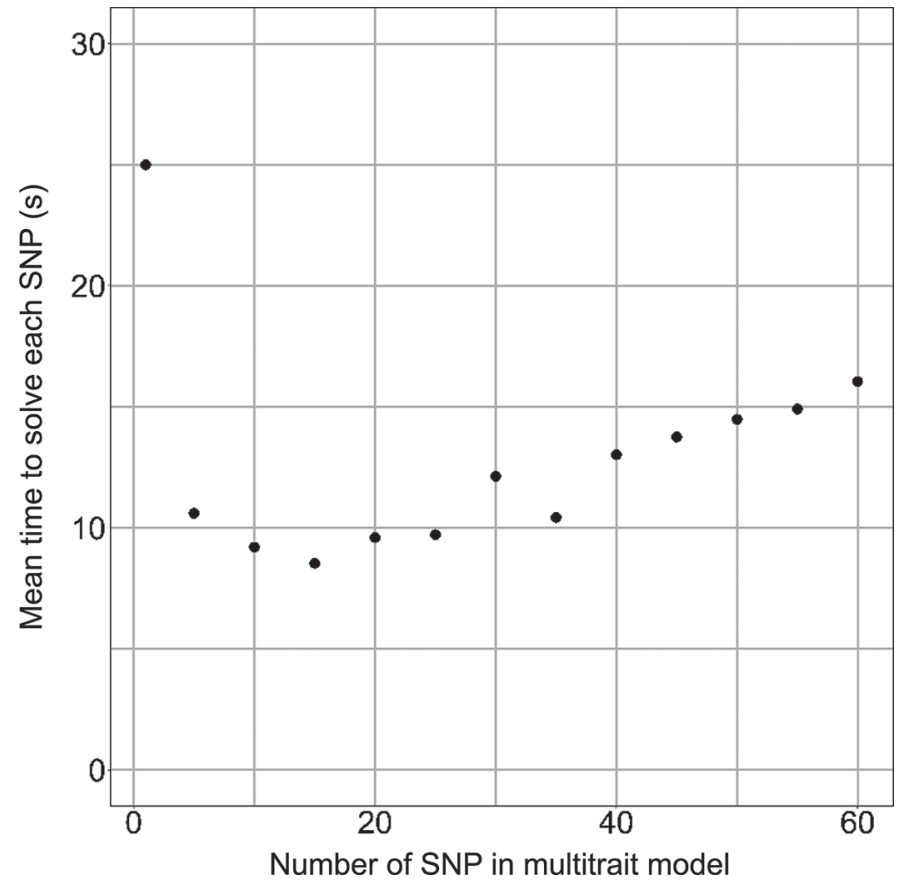

Figure 2. Mean time per SNP for MiXBLUP (Ten Napel et al., 2017) to start and end, solving mixed model equations, with the base simulation data set.

GLS_Sparse methods. For the computation of $\mathbf{A}_{22}^{-1}$ with Calc_grm, 1 node with 64 cores, 1-TB memory, AMD Opteron, and $2.3 \mathrm{GHz}$ was used. A total of 16 threads were used for Calc_grm, but the implementation of the full $\mathbf{A}_{22}^{-1}$ in $\hat{\mu}_{i}=\left(\mathbf{1}^{\prime} \mathbf{A}_{22}^{-1} \mathbf{1}\right)^{-1} \mathbf{1}^{\prime} \mathbf{A}_{22}^{-1} \mathbf{z}_{\mathrm{i}}$ was done with a single thread on the same nodes as the other methods.

To determine whether one of the methods of estimating base generation allele frequency should be used to replace the current method, the method needs to be efficient and at least as accurate. To determine efficiency, both the wall clock time and total processing time were compared between methods. Wall clock time varied depending on the number of central processing units used, whether parallel processing was used, and whether the process had been optimized. That is why it was also important to consider the processing time, which accounts for the total time used across all central processing units and processes. Similarly, for computational efficiency, the total random access memory (RAM) used was also reported to compare memory requirements. Wall clock time, total processing time, and total RAM were recorded as the maximum job requirements, as reported by the HPC. Accuracy was determined by the correlation of the known base generation allele frequency from QMSim and the estimated allele frequency.

\section{RESULTS}

The results for efficiency are presented only for the base simulation without selection because the results were similar for the other simulations (Table 2). We observed that the current method of estimating base generation allele frequency using all genotyped animals was fast (3 s). Using the same method but with only animals from the oldest genotyped generation was even faster (1 s). Using the GLS method with GLS_Sparse required more time, but we still considered it to be efficient (49 s). Methods BLUP (35 min) or the full $\mathbf{A}_{22}^{-1}$ with GLS_Full ( $>1$ d) were not efficient compared with the current method. Finally, the GLS_Sparse method was also tested with the 50K SNP data set, which required 6 min of processing time.

The processing time for the current method and only the oldest genotyped generation had no additional time requirements compared with the wall clock time. The GLS_Sparse method was the fastest alternative method $(49 \mathrm{~s})$. The total processing time for the BLUP analysis

Table 2. Computational time (day-h:min:s) and memory requirements to complete the full process of each method for the base simulation without selection

\begin{tabular}{lrcr}
\hline Method & Process time & Wall clock time & $\begin{array}{c}\text { Random access } \\
\text { memory (GB) }\end{array}$ \\
\hline Current method & $0-00: 00: 03$ & $0-00: 00: 03$ & $<0.1$ \\
Oldest genotyped animals $_{111 \text { MiXBLUP }}^{1}$ & $0-00: 00: 01$ & $0-00: 00: 01$ & $<0.1$ \\
${\text { 1 MiXBLUP of } 15 \text { SNP }^{2}}_{\text {GLS_Sparse }^{3}}$ & $0-12: 42: 47$ & $0-00: 10: 50$ & 48.9 \\
GLS_Full $^{4}$ & $0-00: 06: 20$ & $0-00: 06: 20$ & 0.4 \\
\hline
\end{tabular}

${ }^{1}$ Requirements for 111 BLUP runs including 15 SNP and 1 run including 5 SNP (MiXBLUP software; Ten Napel et al., 2017).

${ }^{2}$ Average requirements for 111 BLUP runs including 15 SNP.

${ }^{3}$ General least squares (GLS) method using sparse matrices.

${ }^{4}$ GLS method using dense matrices. 
(12 h $42 \mathrm{~min}$ ) was a cumulative amount of time caused by the total number of individual runs required in MiXBLUP of 15 correlated SNP (minimum time per run $<5$ min). Less than $10 \mathrm{~s}$ per SNP was required for MiXBLUP runs with between 5 and 20 SNP. For a run with 60 SNP, approximately 15 s per SNP was required during solving (Figure 2). The total processing time was increased for $60 \mathrm{SNP}$ (13 h $42 \mathrm{~min}$ ) due to the minimum time per run $(\sim 30 \mathrm{~min})$, but there was no significant difference in memory requirements. The total processing time for the GLS method using the full $\mathbf{A}_{22}^{-1}$ was exceptionally demanding ( $>19$ d), the majority of which was used to invert the $\mathbf{A}_{22}$ matrix using Calc_ grm.

The total RAM required for each method was closely related to the total processing time (Table 2 ). The current method required very little memory $(<0.1 \mathrm{~GB})$ and required even less when using only the oldest genotyped generation $(<0.1$ GB). GLS_Sparse required more RAM (1.3 GB) but was still computationally efficient. When the 50K SNP data set was used, GLS_ Sparse required up to 37.6 GB. The RAM requirements for the BLUP analysis with 1,670 SNP was large (49.0 $\mathrm{GB})$ due to the individual BLUP runs of $15 \mathrm{SNP}$, which required $0.4 \mathrm{~GB}$ each. Using the full $\mathbf{A}_{22}^{-1}$ for the GLS validation was the most demanding (118.2 GB), again primarily due to storing the full $\mathbf{A}_{22}^{-1}$ matrix and its inverse with Calc_grm (78.4 GB).

For all data sets and methods, there was no significant difference in accuracy between the full 1,670 SNP and the subsets of 33 independent SNP; therefore, only the results for the full data sets are presented. When using the base simulation with no selection, the accuracies, computed as correlations between the estimated allele frequency and the known simulated frequency, were not different from $1(0.99 \pm 0.01)$ for all methods (Table 3). Significant differences in accuracy between methods were observed for simulations that included selection. When using the current method with all genotyped animals, the accuracy decreased to $0.87 \pm 0.01$; using only the oldest genotyped generation, the accuracy was slightly increased but was not significantly different $(0.88 \pm 0.01)$. We observed that both the BLUP (0.94-0.97) and GLS (0.93-0.97) methods significantly increased the accuracy for all data simulations under selection. There was no significant difference between the BLUP and GLS methods, with a correlation of 0.99 \pm 0.01 observed with the base simulation under selection (Figure 3). For both the BLUP and GLS methods, the estimated allele frequencies were more similar to the true base generation allele frequency for allele frequencies $<0.10$ and $>0.90$, whereas larger differences were observed in which the true allele frequency was closer to 0.50 (Figure 4).

When founders were erroneously identified in the pedigree between generations 1 and 5 , the accuracy was still improved with both BLUP $(0.94 \pm 0.01)$ and GLS $(0.93 \pm 0.01)$ compared with the current method (0.87 $\pm 0.01)$. When the incomplete pedigree was continued up to generation 8 , the accuracy was decreased for the BLUP and GLS methods $(0.91 \pm 0.01)$. The accuracy with the incomplete pedigree was lower compared with the other data simulations. Introducing $5 \%$ missing genotypes or $5 \%$ genotyping errors did not affect the accuracy $(0.97 \pm 0.01)$. When both the $5 \%$ missing and $5 \%$ genotyping errors were included, none of the methods were affected. The missing genotype rate was reanalyzed for the GLS_Sparse method to see what effect different missing genotype rates (between 1 and $10 \%$ ) had on the accuracy of estimation (Figure 5). The GLS_Sparse method was very robust; even up to $10 \%$ missing genotypes the accuracy was not significantly different from 0.97 , although the accuracy did start to decrease after $8 \%$ missing genotypes (0.96).

Table 3. Correlations between the known base generation allele frequency and estimated allele frequency (all SE $<0.01)$

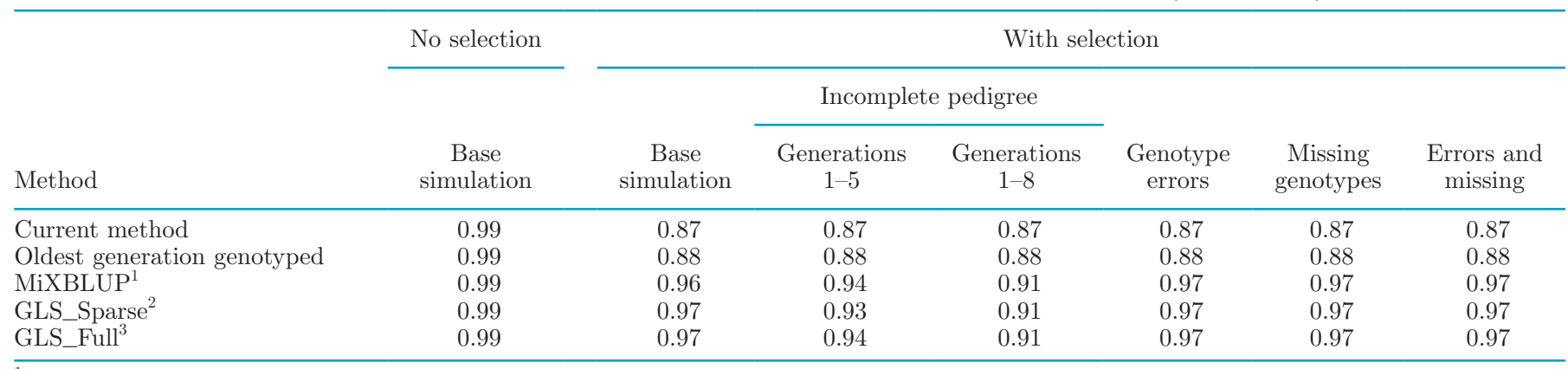

${ }^{1}$ MiXBLUP software (Ten Napel et al., 2017).

${ }^{2}$ General least squares (GLS) method using sparse matrices.

${ }^{3}$ GLS method using dense matrices. 


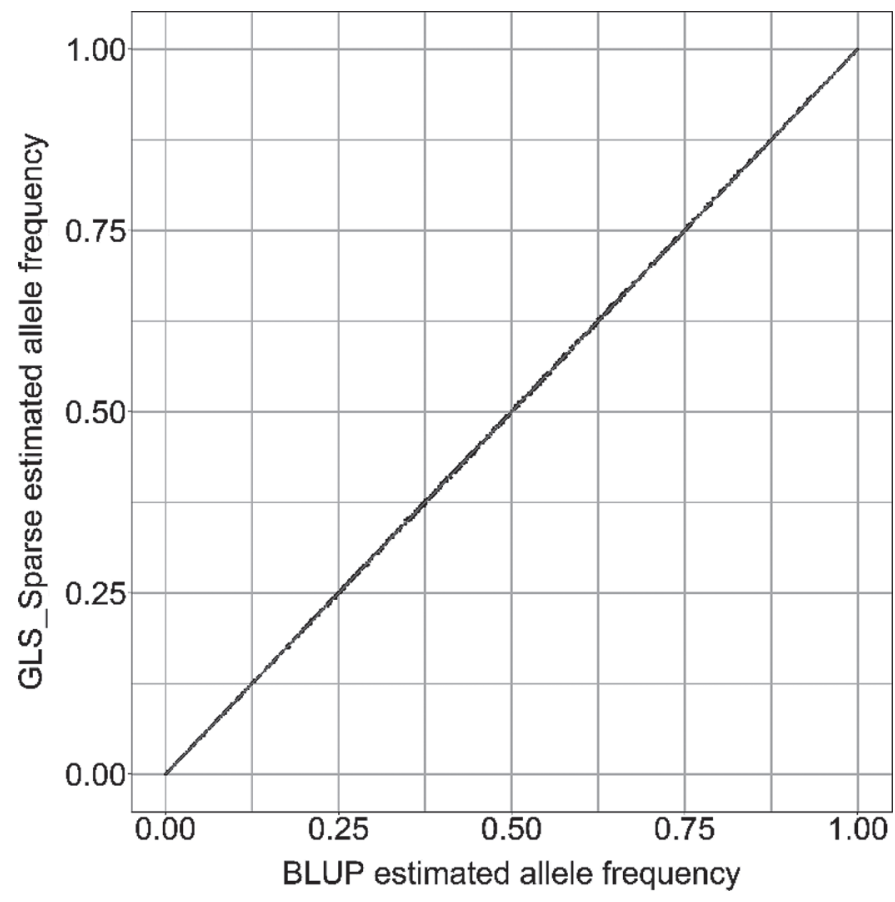

Figure 3. The allele frequency estimated with BLUP versus GLS_ Sparse (general least squares method using sparse matrices) for the base simulation with selection.

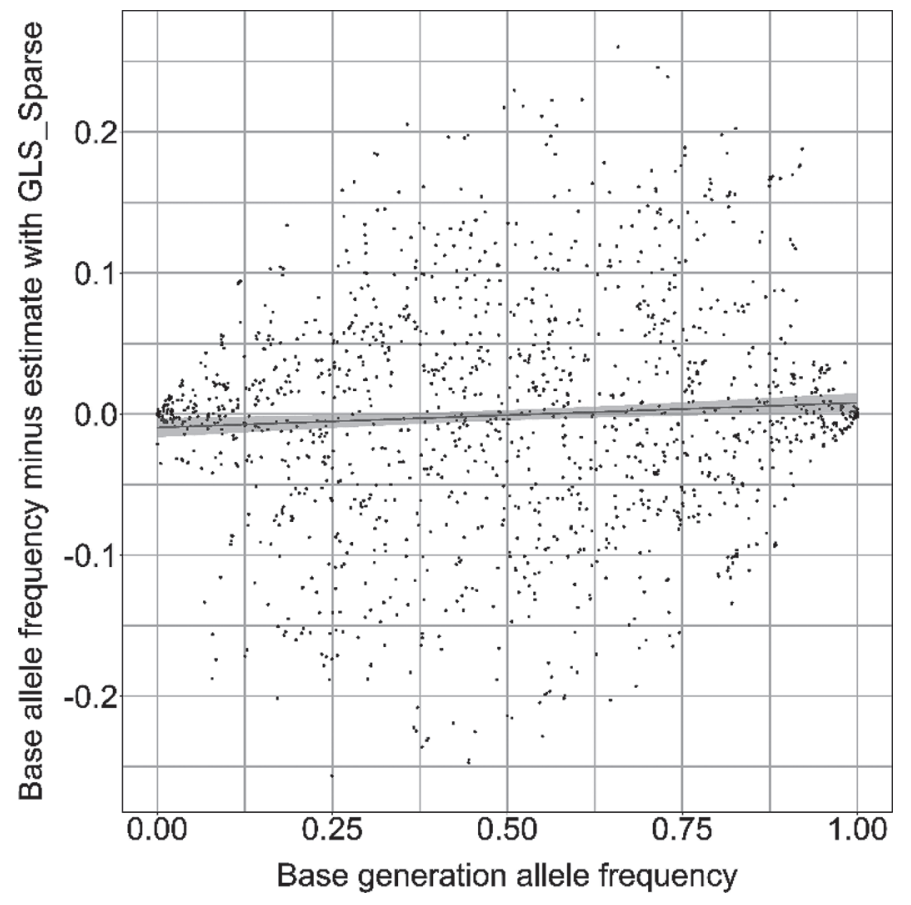

Figure 4. The relationship between the base generation allele frequency, and the difference between the estimated allele frequency with GLS_Sparse (general least squares method using sparse matrices) compared with the base generation allele frequency, with a linear regression and $95 \%$ CI, for the base simulation with selection.

\section{DISCUSSION}

The objective of this study was to compare methods for estimating base generation allele frequencies in terms of efficiency and accuracy. The only method that was both efficient and accurate was the GLS method using GLS_Sparse. With wall clock and processing times of less than $1 \mathrm{~min}$, GLS_Sparse can be implemented in routine genomic evaluations without jeopardizing overall efficiency. The RAM requirements for the GLS_Sparse are linearly related to the number of SNP, as shown by the results obtained with the 50K SNP data set. Although the time requirement is already limited ( $<10$ min for the $50 \mathrm{~K}$ data set), it could be even further improved by using parallel processing because the MKL library and Pardiso are multithreaded. For example, the wall clock time was reduced to less than 5 min when using 4 threads. The 50K SNP was not analyzed with the BLUP method but would require 3,340 runs of 15 SNP each. Assuming that each run was equal to the mean time required (0-00:06:20), the required processing time would be more than $14 \mathrm{~d}$, and the observed wall clock time would be limited by the number of parallel MiXBLUP runs that can be run at the same time. As already demonstrated, the GLS_Full was already inefficient for 1,670 SNP, and no attempt to ana-

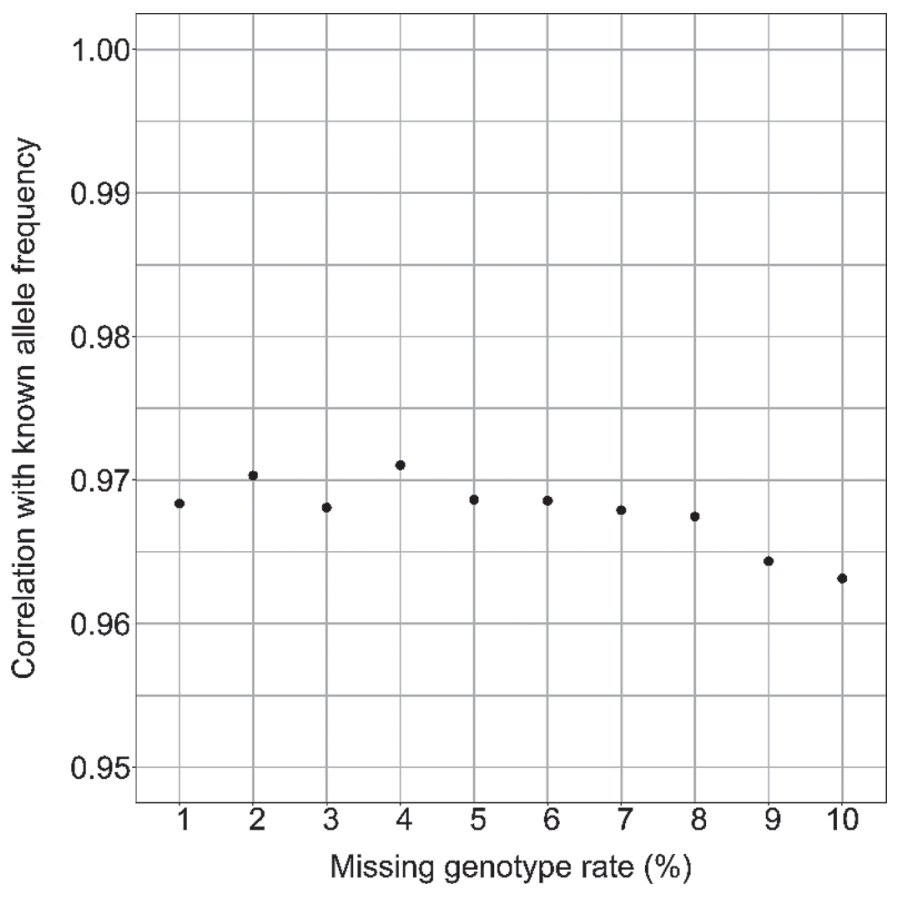

Figure 5. The relationship between increasing the missing genotype rate and the correlation between estimated frequency with GLS_ Sparse (general least squares method using sparse matrices) and the known base allele frequency. 
lyze the 50K SNP data set with GLS_Full was made, nor is it recommended. It is worth noting that computing explicitly $\mathbf{A}_{22}^{-1}$ is not strictly necessary for GLS_Full because we need the product $\mathbf{t}=\mathbf{A}_{22}^{-1} \mathbf{1}$, which can be more efficiently computed as $\mathbf{t}=\mathbf{L}^{-1},\left[\mathbf{L}^{-1} \mathbf{1}\right]$, with the matrix $\mathbf{L}$ being the Cholesky factor of $\mathbf{A}_{22}$. This strategy would request the same amount of memory as GLS_Full and will be considerably faster than GLS Full. Even then it would still be computationally much less efficient than GLS_Sparse.

Importantly, GLS_Sparse is more accurate than the current method that simply computes the allele frequency in the current genotype data. It is recommended that the GLS_Sparse method be implemented when using allele frequencies for genomic prediction processes, where the assumption requires base generation frequencies. Arguably, with increasing amounts of genotype data available, the estimated base generation allele frequencies will not change as much over time as the allele frequencies in the genotype data. In practical implementations, one could consider not re-estimating base generation allele frequencies for every run of the genetic evaluation. Instead, they could be re-estimated, for instance, every time the variance components of the model are re-estimated. Any possible fluctuations in results (e.g., genomic EBV) caused by changing allele frequencies when new genotyped animals are added and when using the current method would therefore occur only when the frequencies are re-estimated and not for every evaluation.

There was no significant difference in accuracy between the GLS and BLUP methods because both use the pedigree information. Both GLS and BLUP had high correlations with the known base generation allele frequencies; estimates are virtually the same with incomplete pedigree, but the estimates from the 2 methods were different with both genotyping errors and missing genotype data sets. Additional analyses with BLUP (results not shown), mimicking the GLS implementation by using a heritability of 0.99999 and replacing missing genotypes by the average genotype in the data, confirmed that the difference between GLS and BLUP is due to using a nonunity heritability in BLUP and to replacing genotypes in GLS with the average (which is probably worse than putting it to missing in BLUP). However, in many practical applications, replacing missing values in the GLS method will probably be unnecessary because imputation is common practice. When a considerable number of genotyping errors are present, the BLUP method may be better able to deal with this, as it has been suggested to be robust against genotyping errors (Gengler et al., 2007). In such cases the heritability used should probably re- flect the proportion of genotyping errors, and a value lower than our value of 0.99 may be more appropriate. In fact, the heritability of the genotypes of each SNP could be estimated to assess its quality in the first place (Forneris et al., 2015).

Results for the simulated scenario with selection did indicate that estimated allele frequencies deviated considerably, up to 0.25 unit, from the actual values. Observed deviations were larger for allele frequencies closer to 0.50 and limited at $<0.10$ or $>0.90$. This is because the estimates of allele frequencies closer to 0 or 1 were on one side bounded to stay within the parameter space. The simulations used were rather extreme in the sense that changes in allele frequencies up to 0.7 unit were observed across 12 generations of selection. In real-life breeding programs it is unlikely to see so many loci with such big changes in allele frequencies in such a short time frame, so the expected deviations of the estimated from the true base allele frequencies are expected to be smaller.

The only partial limitation observed with the GLS Sparse method was that SNP that had a minor allele frequency below 0.001 in both the base generation and current population would sometimes result in an estimate outside of the parameter space. This was also observed with the BLUP method. Three SNP in the base simulation without selection were outside of the parameter space (outside parameter space by $<0.001$ ). Similar numbers of SNP were observed outside the parameter space for the other data simulations. These SNP also had known minor allele frequencies in the base generation of $<0.001$ and known minor allele frequencies in generation 12 of $<0.001$. Such estimates have been observed by VanRaden (2008) and Makgahlela et al. (2013), which suggested that these outliers are due to the use of linear algebra instead of nonlinear probabilities.

Alternatively, the current method was used to filter SNP with a minor allele frequency $(<0.01)$ before running GLS_Sparse. The only benefit was that it did remove the SNP with estimates that previously were outside the parameter space (results not shown). Realistically, those SNP would be removed during standard processing practices before being used in GLS_Sparse, and estimates outside the parameter space are not expected to occur. If the base allele frequency is needed for all markers, it may be necessary to assume that they are fixed by assigning missing or zero to markers outside the parameter space. We conclude that the GLS_Sparse method is efficient, robust, and accurate within the range of allele frequencies 0.01 to 0.99 .

When animals were erroneously identified as founders due to incomplete pedigree, we observed a signifi- 
cant decrease in accuracy for the BLUP and GLS methods. The accuracy was decreased further when removing the pedigree for $25 \%$ of the animals up until generation 8 , which was the last nongenotyped generation. This effectively meant that animals from later generations were added to the base, and because allele frequencies changed across generations, the estimates represented some sort of average across generations instead of those in the base generation. It is important to note that the accuracy for the BLUP and GLS methods was still greater compared with the current method. The accuracy for such cases could be improved by taking into account the different base populations by implementing the GLS_Sparse method with genetic groups. This could be done by replacing the vector $\mathbf{1}$ in the different formulae by a matrix $\mathbf{Q}$ that contains the expected fraction of each genetic group for each genotyped individual-that is, $\hat{\boldsymbol{\mu}}_{i}=\left(\mathbf{Q}^{\prime} \mathbf{A}_{22}^{-1} \mathbf{Q}\right)^{-1} \mathbf{Q}^{\prime} \mathbf{A}_{22}^{-1} \mathbf{z}_{i}$, with $\hat{\boldsymbol{\mu}}_{i}$ being a vector of estimates of base allele frequencies for all genetic groups (Gengler et al., 2007; VanRaden, 2008; Makgahlela et al., 2013; Garcia-Baccino et al., 2017). The strategies used for GLS_Sparse are readily extendable for the computation of $\mathbf{Q}^{\prime} \mathbf{A}_{22}^{-1}$ and $\left(\mathbf{Q}^{\prime} \mathbf{A}_{22}^{-1} \mathbf{Q}\right)^{-1}$.

Allele frequencies are required for several processes in genomic prediction. This includes computation of model-based reliabilities of direct genomic values in the context of multistep genomic evaluations, computation of genomic relationships to be used in single-step genomic BLUP, computation of relationships among metafounders, and compatibility between the pedigree and genomic relationship matrices. The bias due to compatibility between the relationship matrices can be overcome by adjusting the genomic relationship by blending with the pedigree matrix (Gao et al., 2012) or shifting the genomic relationships by an analytically derived constant (Vitezica et al., 2011). Alternatively, the pedigree relationship matrix can be adjusted by scaling it to the genomic relationship matrix (Christensen, 2012). Although these adjustments for the relationship matrices could be more efficient than the computation of base allele frequencies when performing a genomic evaluation, it can be assumed that the computation of base allele frequencies could be performed only once for multiple successive genomic evaluations (e.g., at the same rate as variance components estimation), which would reduce its costs even further.

\section{CONCLUSIONS}

There are several benefits for calculating base generation allele frequencies using the general least squares method, with a pedigree relationship matrix computed using sparse matrices. It is fast; therefore, practical application is appropriate and would not delay other processes. It is accurate in estimating base generation allele frequencies under several different scenarios, thereby better fulfilling the assumptions of genomic prediction processes than the current method. We recommend that base generation allele frequencies be estimated using a GLS method implemented with sparse matrices for $\mathbf{A}_{22}^{-1}$ and replacing any missing genotypes with the mean allele frequency calculated from the genotyped population or with imputed values.

\section{ACKNOWLEDGMENTS}

This study was financially supported by the Dutch Ministry of Economic Affairs (TKI Agri \& Food project 16022) and the Breed4Food partners Cobb Europe (Colchester, Essex, United Kingdom), CRV (Arnhem, the Netherlands), Hendrix Genetics (Boxmeer, the Netherlands), and Topigs Norsvin (Helvoirt, the Netherlands). The use of the high-performance cluster was made possible by CAT-AgroFood (Shared Research Facilities Wageningen UR, Wageningen, the Netherlands).

\section{REFERENCES}

Aguilar, I., I. Misztal, D. Johnson, A. Legarra, S. Tsuruta, and T. Lawlor. 2010. Hot topic: A unified approach to utilize phenotypic, full pedigree, and genomic information for genetic evaluation of Holstein final score. J. Dairy Sci. 93:743-752. https://doi.org/10 .3168/jds.2009-2730.

Calus, M., and J. Vandenplas. 2016. Calc_grm-A Program to Compute Pedigree, Genomic, and Combined Relationship Matrices. ABGC, Wageningen UR Livestock Research, Wageningen, the Netherlands.

Christensen, O. F. 2012. Compatibility of pedigree-based and marker-based relationship matrices for single-step genetic evaluation. Genet. Sel. Evol. 44:37. https://doi.org/10.1186/1297-9686-44-37.

Christensen, O. F., and M. S. Lund. 2010. Genomic prediction when some animals are not genotyped. Genet. Sel. Evol. 42:2. https:// doi.org/10.1186/1297-9686-42-2.

Daetwyler, H. D., M. P. Calus, R. Pong-Wong, G. de los Campos, and J. M. Hickey. 2013. Genomic prediction in animals and plants: Simulation of data, validation, reporting, and benchmarking. Genetics 193:347-365. https://doi.org/10.1534/genetics.112.147983.

Forneris, N. S., A. Legarra, Z. G. Vitezica, S. Tsuruta, I. Aguilar, I. Misztal, and R. J. Cantet. 2015. Quality control of genotypes using heritability estimates of gene content at the marker. Genetics 199:675-681. https://doi.org/10.1534/genetics.114.173559.

Gao, H., O. F. Christensen, P. Madsen, U. S. Nielsen, Y. Zhang, M. S. Lund, and G. Su. 2012. Comparison on genomic predictions using three GBLUP methods and two single-step blending methods in the Nordic Holstein population. Genet. Sel. Evol. 44:8. https://doi .org/10.1186/1297-9686-44-8.

Garcia-Baccino, C. A., A. Legarra, O. F. Christensen, I. Misztal, I. Pocrnic, Z. G. Vitezica, and R. J. Cantet. 2017. Metafounders are related to $\mathrm{F}_{\text {st }}$ fixation indices and reduce bias in single-step genomic evaluations. Genet. Sel. Evol. 49:34. https://doi.org/10 .1186/s12711-017-0309-2.

Gengler, N., P. Mayeres, and M. Szydlowski. 2007. A simple method to approximate gene content in large pedigree populations: Appli- 
cation to the myostatin gene in dual-purpose Belgian Blue cattle. Animal 1:21-28. https://doi.org/10.1017/S1751731107392628.

Henderson, C. R. 1984. Applications of Linear Models in Animal Breeding. 2nd ed. University of Guelph, Guelph, ON, Canada.

Legarra, A., O. F. Christensen, Z. G. Vitezica, I. Aguilar, and I. Misztal. 2015. Ancestral relationships using metafounders: Finite ancestral populations and across population relationships. Genetics 200:455-468. https://doi.org/10.1534/genetics.115.177014.

Makgahlela, M. L., I. Strandén, U. Nielsen, M. Sillanpää, and E. Mäntysaari. 2013. The estimation of genomic relationships using breedwise allele frequencies among animals in multibreed populations. J. Dairy Sci. 96:5364-5375. https://doi.org/10.3168/jds.2012-6523.

McPeek, M. S., X. Wu, and C. Ober. 2004. Best linear unbiased allelefrequency estimation in complex pedigrees. Biometrics 60:359-367. https://doi.org/10.1111/j.0006-341X.2004.00180.x.

Mrode, R. 2005. Appendix C. Pages 303-306. Linear Models for the Prediction of Animal Breeding Values. 2nd ed. CABI Publishing, Wallingford, UK.

Sargolzaei, M., and F. S. Schenkel. 2009. QMSim: A large-scale genome simulator for livestock. Bioinformatics 25:680-681. https:// doi.org/10.1093/bioinformatics/btp045.
Schenk, O., K. Gärtner, W. Fichtner, and A. Stricker. 2001. PARDISO: A high-performance serial and parallel sparse linear solver in semiconductor device simulation. Future Gener. Comput. Syst. 18:69-78. https://doi.org/10.1016/S0167-739X(00)00076-5.

Strandén, I., K. Matilainen, G. Aamand, and E. Mäntysaari. 2017. Solving efficiently large single-step genomic best linear unbiased prediction models. J. Anim. Breed. Genet. 134:264-274. https:// doi.org/10.1111/jbg.12257.

Ten Napel, J., J. Vandenplas, M. Lidauer, I. Stranden, M. Taskinen, V. Mäntysaari, M. P. L. Calus, and R. F. Veerkamp. 2017. MiXBLUP, user-friendly software for large genetic evaluation systems-Manual. Accessed Oct. 10, 2017. http://www.mixblup.eu/ documents/Manual\%20MiXBLUP\%202.1_June\%202017_V2.pdf.

VanRaden, P. M. 2008. Efficient methods to compute genomic predictions. J. Dairy Sci. 91:4414-4423. https://doi.org/10.3168/jds .2007-0980.

Vitezica, Z. G., I. Aguilar, I. Misztal, and A. Legarra. 2011. Bias in genomic predictions for populations under selection. Genet. Res. (Camb.) 93:357-366. https://doi.org/10.1017/ S001667231100022X 